\title{
POPULISMUS IN KOLUMBIEN: DIE ALIANZA NACIONAL POPULAR (ANAPO)
}

\author{
Von W. Wessel-Schulze
}

\section{Die Tradition des Zwei-Parteien-Systems}

Seit dem Ende des Unabhängigkeitskrieges im Jahre 1821 gibt es zwei große Parteien in Kolumbien. Im 19. Jahrhundert repräsentierten sie unterschiedliche Staatsauffassungen. Die Konservative Partei vertrat die Idee des Zentralismus, die Liberale Partei die des Föderalismus. Mit der Idee des Föderalismus ging die liberale Auffassung der Trennung von Staat und Kirche einher und war eine der wichtigsten Forderungen, um die die beiden Parteien jahrzehntelang stritten. Diese Auseinandersetzungen wurden mit aller politischen Härte, ja sogar militärisch geführt und fanden ihren Höhepunkt im Bürgerkrieg von 1899-1902.

Bis zum Jahre 1948 hatte jeweils eine der beiden Parteien über mehrere Jahrzehnte regiert. Die liberale Parteiherrschaft begann 1849. Wesentliche Punkte ihres Parteiprogramms brachte sie in die Verfassung von 1863 ein. Als 1880 die Konservative Partei die Macht übernahm, machte sie all diese Änderungen rückgängig und sorgte mit Hilfe der Verfassung von 1886 für die Wiederherstellung der alten Ordnung. Die Konservativen beherrschten bis 1934 die politische Bühne.

Die grundlegenden Gegensätze zwischen den Liberalen und Konservativen in politischen, wirtschaftlichen und religiösen Auffassungen wurden erst im 20. Jahrhundert langsam abgebaut. Gemeinsam war ihnen stets, daß eine kleine aristokratische Elite die Partei und die Politik bestimmte. Diese Parteieliten waren flexibel genug, um Mitglieder der im 20. Jahrhundert entstehenden Mittelschicht aufzunehmen. Die breite Unterschicht stand immer am Rande oder außerhalb des politischen Geschehens. Doch die Sympathie des Volkes zu einer der beiden Parteien war stets vorhanden und implizierte tiefverwurzelte Vorurteile, und Feindseligkeiten gegenüber der gegnerischen Partei.

Miguel Antonio Caro sieht deshalb in den Parteien weniger politische Organisationen als vielmehr den Ausdruck von „ererbtem Haß"1. Dieser Parteienhaß hat die Politik im 19. aber auch im 20. Jahrhundert erneut geprägt. In den Jahren 1946-1958 fand er seinen Ausdruck in der bürgerkriegsähnlichen „violencia“2, in dem mit unvorstellbaren Grausamkeiten ausgetragenen Parteienkampf. Seit 1958 wird die violencia von der Tätigkeit linker Guerillagruppen bestimmt.

Erst nach der Militärdiktatur des Generals Rojas Pinilla fanden sich die beiden Parteien bereit, gemeinsam und in gesetzlich geregelter Teilung aller politischer Ämter miteinander zu regieren. Der Präsident wurde in ständigem Wechsel von einer der beiden Parteien gestellt. Diese Parteienkoalition, die „Frente Nacional“ (Nationale Front), hat seit 1958 für politische Stabilität gesorgt.

1 Zitiert bei: R. H. Dix, Colombia: The Political Dimensions of Change, New Haven/London 1969 2, S. 211.

2 Einen umfassenden Uberblick gibt die soziologische Untersuchung von: G. Guzmán/O. Fals Borda, E. Umana Luna "La Violencia en Colombia“, in: Monografias Sociologicas, Nr. 12, Vol. 1, Bogota 1962; R. C. Williamson, "Toward a Theory of Political Violence: The Case of Rural Colombia", in: The Western Political Quaterly, 18. Jg., March 1965, S. 35-44; Dix, a. a. O., S. 360-382. 
Die Wahlbeteiligung ist seit 1958 von Wahl zu Wahl geringer geworden ${ }^{3}$, denn eine politische Änderung konnte nicht eintreten. Die Frente Nacional sollte 16 Jahre dauern, ist aber inzwischen bis 1978 für die Wahlen der Gouverneure und Vertreter in den Rathäusern verlängert worden, wogegen sich allerdings die übrigen Parteien ausgesprochen und ein baldiges Ende gefordert haben ${ }^{4}$.

Kolumbien konnte bis 1971 als die Repräsentation eines traditionellen Parteiensystems in Lateinamerika gelten. Ein Prunkstück der sogenannten „politischen Museen" dieser Region". Mit der Parteigründung der Alianza Nacional Popular (ANAPO) 1971 trat erstmals eine zahlenmäßig bedeutende dritte Partei auf. Die Parteien der Kommunisten und Sozialisten waren stets zu klein gewesen, um eine Gefahr für die Macht der traditionellen Parteien darzustellen.

\section{Die Diktatur des Rojas Pinilla}

1953 hatte der General Rojas Pinilla als Oberbefehlshaber aller Streitkräfte den Höhepunkt seiner militärischen Karriere erreicht. Er vertrat die traditionelle Auffassung des Militärs, daß es sich aus der Politik herauszuhalten und für die Sicherheit und Ordnung im Lande zu sorgen habe. Das kolumbianische Militär hatte, im Gegensatz zu den meisten anderen lateinamerikanischen Ländern, nur dreimal seit der Unabhängigkeit von Spanien in die Politik eingegriffen: 1830, 1854, $1900^{6}$.

Die „violencia"7, die hauptsächlich im Landesinnern auf dem Rücken der Bauern ausgetragen wurde, brachte das Land mit zunehmender Dauer an den Rand des Chaos. Der seit 1950 regierende konservative Präsident, Laureano Gómez, unterstützte mit diktatorischen Maßnahmen seine Partei und verstärkte hierdurch den schwelenden Bürgerkrieg. Das Militär sympathisierte zwar mit den Konservativen, konnte aber der ständig zunehmenden Willkür gegenüber den Liberalen und dem Verfall der Staatsordnung nicht länger tatenlos zusehen. Außerdem sah Gómez zu Unrecht in Rojas einen politischen Rivalen, den er mehrmals durch Intrigen aus seinem Amt zu verdrängen suchte ${ }^{8}$. Als er in letzter Konsequenz den Oberbefehlshaber der Streitkräfte und den Kriegsminister ablösen lassen wollte, putschte das Militär unter der Führung von Rojas Pinilla am 13. Juni 1953.

Rojas hatte bei der Machtübernahme betont, daß das Militär die Macht an zivile Kräfte zurückgeben werde, wenn Friede, Sicherheit und Ordnung wiederhergestellt wären. Obwohl diese Bedingungen 1954 erfüllt waren, ließ er sich im selben Jahr zum Präsidenten wählen.

Die stark nationalistisch und christlich gefärbte Ideologie des Regimes verfolgte das Ziel, die Lebensbedingungen der unterprivilegierten Kolumbianer zu verbessern'. Rojas ließ Straßen, Flugplätze Schulen etc. bauen, verstaatlichte die AVIANCA Fluggesellschaft und gründete die staatliche Erdölgesellschaft ECOPETROL. Er verbesserte das Kreditsystem für die kleinen Bauern und führte das

\footnotetext{
3 Vgl. P. Gilhodes, „L'Abstention Electorale en Colombie - Les Elections de Mars 1968“, in: Notes et Etudes Documentaires, 21 Avril 1969, Nr. 12, Nos 3582-3583, S. 56-67.

4 M. Neubert, "Tauwetter für Kolumbiens eingefrorene Demokratie “, in: Die Welt, 15. August 1973.

$5 \mathrm{Kl}$. Lindenberg, "Politische Parteien in Lateinamerika“ in: W. Grabendorff (Hrsg.) Lateinamerika Kontinent in der Krise, Hamburg 1973, S. 141-142.

6 Dix, a. a. O., S. 295.

7 Sie forderte in der Zeit von 1946-1958 zwischen 100000 und 300000 Todesopfer. Die Angaben schwanken erheblich. Vgl. Guzmán/Fals Borda, La Violencia, a. a. O. Dix; a. a. O., S. 362-386.

8 Vgl. J. D. Martz, Colombia, Chapel Hill 1962, S. $162 \mathrm{ff}$.

9 S. Pope Brewer, „Bogotá News Curb Ordered Removed“, in: New York Times, 20. August 1953, S. 13.
} 
Wahlrecht für Frauen ein. Erstmals in der Geschichte Kolumbiens wurden die Aktiendividenden besteuert, damit verstärkte sich aber gleichzeitig die Opposition der Oligarchie. Das Regime trug eindeutig sozialreformerische Züge, war aber gegenüber Kritik äußerst empfindlich.

Mit dem Verbot der beiden Tageszeitungen „El Tiempo“ und „El Espectador“, 1955, die als Sprachrohr der beiden Parteien Rojas kritisiert und attackiert hatten, begann die diktatorische Phase des Regimes. War die militärische Ordnung zuerst die Grundlage für Freiheit, Gerechtigkeit und Frieden gewesen ${ }^{10}$, so wurde sie jetzt das Instrument der Unterdrückung der Freiheit. Demonstrierende Studenten wurden vom Militär niedergeschossen, angebliche Regimegegner in der Stierkampfarena 1956 niedergeknüppelt und getötet.

Die anfängliche Unterstützung der beiden Parteien verringerte sich zusehends und veranlaßte Rojas Pinilla zur Gründung einer Volksbewegung, die als „Dritte Kraft“ über den Parteien stehen sollte. Die feierliche Gründung am 17. Juni 1956 war zugleich Anfang und Ende dieser Bewegung. Besonders die katholische Kirche, die eine solide Stütze seiner Macht war, hatte sich in schärfster Form dagegen geäußert, weil sie in der Vereinigung der Gewerkschaft Confederacion Nacional Trabajadores (CNT) mit der "Dritten Kraft" eine Konkurrenz zu der von ihr unterstützten Union de Trabajadores Colombianos (UTC) sah.

Die Diktatur von Rojas Pinilla hatte die Konservative und Liberale Partei durch ihre gemeinsame Ablehnung des Regimes und die Furcht vor dem Abbau ihrer politischen und wirtschaftlichen Privilegien wieder zusammengebracht. Die beiden Parteiführer trafen sich 1956 in Spanien. Sie vereinbarten, den Sturz von Rojas Pinilla zu betreiben und danach eine auf 16 Jahre befristete Regierungskoalition zu bilden. Im Frühjahr 1957 hatte Rojas jede politische Unterstützung verloren, auch im Militär und in der katholischen Kirche. Die Straßenkämpfe zwischen Soldaten und Demonstranten waren alltäglich geworden und die Wirtschaft zerrüttet. Im Mai zwang ihn dasselbe Militär, das ihn an die Macht gebracht hatte, zurückzutreten. Einige Monate regierte eine Militärjunta, bis die Parteienkoalition aus Konservativen und Liberalen, die „Frente Nacional“, die Macht übernahm.

Der Exdiktator ging ins Exil. Seine frühe Rückkehr im Oktober 1958 war mit der Hoffnung verbunden, erneut Unterstützung bei einem großen Teil des Militärs und der Bevölkerung zu finden. Darauf aufbauende Putschversuche scheiterten, doch veranlaßten sie den Senat, sich als Gericht zu konstituieren und Rojas nachträglich den Prozeß wegen Bereicherung im Amte und Entwürdigung des Präsidentenamtes zu machen. Das Ergebnis des langwierigen Prozesses war die Aberkennung seiner Pensionsansprüche und aller politischen Rechte auf Lebenszeit $^{11}$. Seit Ende 1960 wieder auf freiem Fuß, widmete sich Rojas Pinilla der Aufgabe, innerhalb der Konservativen Partei seine noch verbliebenen Anhänger sowie neue um sich zu scharen. So entstand innerhalb dieser Partei ein selbständiger Rojas-Flügel, der in den folgenden Jahren immer stärker wuchs. Auch innerhalb der Liberalen Partei gewann er Anhänger, wenn auch eine geringere Anzahl.

10 Mensajes y Discursos 1954 - Discursos y Mensajes Presidenciales - (Hrsg. Dirección de Información

11 Der Oberste Gerichtshof gab Rojas 1967 alle Rechte zurück. Eine ausführliche Beschreibung des Prozesses in: Rojas Pinilla ante el Senado, Bogotá 1959. 
Die Zahl der Anhänger in den beiden Parteien vergrößerte sich in den sechziger Jahren beständig. Aufgrund der Frente Nacional-Bedingungen konnten die RojasAnhänger nur im Rahmen der Konservativen- und Liberalen Partei politische Aktivität entfalten. Die Erfolge der Rojas-Gruppen innerhalb beider Parteien im letzten Jahrzehnt haben sie ganz eindeutig als eigenständige politische Gruppierung gekennzeichnet. Die Gründung der Alianza Nacional Popular (ANAPO) als eine von den beiden traditionellen Parteien unabhängige Partei ist nur ein letzter Schritt in die politische Legalität gewesen. Die ANAPO ist wiederholt als "populistisch" bezeichnet worden.

\section{Populismus in Lateinamerika}

Als populistische Parteien oder Bewegungen werden allgemein solche bezeichnet, die von den städtischen oder ländlichen arbeitenden Massen unterstützt werden ${ }^{12}$. Ihre Zusammensetzung ist nicht klassenmäßig festgelegt, vielmehr sind sie polyklassistisch und agieren betont demokratisch und antitotalitär ${ }^{13}$. Sie suchen nach gangbaren demokratischen Wegen, um die immer dringender werdenden sozialen Probleme ihrer Länder zu lösen, die von den traditionellen Parteien, der Konservativen und Liberalen Partei, vernachlässigt wurden. Populistische Parteien beschreiten also einen "Dritten Weg“ zwischen den traditionellen und revolutionären Parteien oder Bewegungen. $\mathrm{Zu}$ den letzteren werden vor allem die kommunistischen, sozialistischen und castrischen Parteien gezählt ${ }^{14}$, die einen Umsturz des bestehenden Gesellschaftssystems anstreben. In den Parteiprogrammen bekennen sich die populistischen Parteien $z u$ einer nationalen, antiimperialistischen und sozial-revolutionären Reformpolitik, d. h. auf der „Basis der Traditionen und Erfordernissen des Subkontinents die sozialen und wirtschaftlichen Gegebenheiten tiefgreifend zu verändern und den Bedingungen der modernen Welt anzupassen ${ }^{15}$.“ Die ersten populistischen Bewegungen entstanden im 19. Jahrhundert in Rußland und in den Vereinigten Staaten von Amerika. Ihre politischen Ziele waren gegensätzlich, anarchistisch in Rußland in der Ablehnung des Staates, systemverändernd aber nicht umstürzlerisch in den Vereinigten Staaten ${ }^{16}$. Gemeinsam war ihnen, daß sie sich für mehr Partizipation der Bauern im Staat einsetzten.

Der Populismus in Lateinamerika hat eine Tradition, die bis in die ersten Jahre des 20. Jahrhunderts zurückreicht. Der Colorado-Politiker José Batlle y Ordònez war der erste, der auf demokratischem Wege soziale Reformen einleitete ${ }^{17}$. Während der mexikanischen Revolution von 1910-1917 konnten die campesinos unter der Führung von Zapata angestammte Rechte der Indios und die Rückgabe bzw. Neuverteilung von Land durchsetzen. Fast alle mexikanischen Präsidenten sahen es als eine Verpflichtung an, weiteres Land zu verteilen.

12 T. S. Di Tella, Populism and Reform in Latin America, in: Claudio Véliz (Hrsg.), Obstacles to Change in Latin America, London 1965, S. 47.

13 R. F. Lamberg, Der lateinamerikanische Populismus, Bad Godesberg, Sommer 1966, S. 3

$14 \mathrm{Kl}$. Lindenberg, "Parteien in Lateinamerika“, in: W. Grabendorff (Hrsg.), Lateinamerika-Kontinent in der Krise, Hamburg 1973, S. 141.

15 Lamberg, a. a. O., S. 3.

16 Vgl. P. Worsley, "The Concept of Populism“, in: G. Ionescu/E. Gellner (Hrsg.), Populism - Its National Characteristics, London 1969, S. 220 ff.

17 Weitere Einzelheiten in: Lamberg, a. a. O., S. 86-91. 
Ein entscheidender Einfluß auf alle späteren populistischen Parteien oder Bewegungen ging von der Alianza Popular Revolucionaria Americana (APRA) in Peru aus, die 1924 von Haya de la Torre gegründet wurde.

Seit 1930 können Varianten populistischer Parteien in fast allen Ländern Lateinamerikas nachgewiesen werden ${ }^{18}$. Ihr Entstehen fällt zusammen mit der Weltwirtschaftskrise 1929, dem dadurch ermöglichten vorzeitigen Abrücken von den USA und dem langsamen Aufbau von eigenen Industrien in den dreißiger Jahren.

Historisch können zwei Varianten des Populismus unterschieden werden, der ländliche und der städtische Populismus ${ }^{19}$. Die Entstehung des städtischen Populismus fällt mit dem Beginn der Industrialisierung und dem Wachsen der Städte zusammen.

Die Vielzahl der heute existierenden populistischen Parteien macht eine Aufgliederung notwendig. Ich übernehme hier die Unterteilung, die Klaus Lindenberg 20 vorgenommen hat. Er unterscheidet zwei Gruppen. Erstens die populistischen Parteien, die als Instrument der Machtsicherung von Diktatoren, wie Getulio Vargas in Brasilien und Juan D. Perón Argentinien, dienten. Die populistische Basis dieser Parteien war überwiegend die soziale Schicht unterhalb der Mittelschicht ${ }^{21}$. Zweitens die "national-revolutionären “22 Populisten-Parteien, die gegen die Diktatur kämpfen und für die Demokratie eintreten. In ihrem Programm setzen sich diese Parteien für sozialreformerische Maßnahmen, insbesondere Agrarreformen ein ${ }^{23}$. Die Basis bildet das Kleinbürgertum und die Unterschicht.

$\mathrm{H}$. Kantor ${ }^{24}$ bezieht in seiner allgemeinen Charakterisierung auch einige weitsichtige Industrielle mit ein.

Die Alianza Nacional Popular (ANAPO) ist der letztgenannten Populismus-Gruppe zuzuordnen.

Die populistischen Parteien, die an der Macht waren, wie die Acción Democrática (AD) in Venezuela, die Partido Liberación Nacional (PLN) in Costa Rica, das Movimiento Nacionalista Revolucionario (MNR) in Bolivien und die Partido Revolucionario Guatemalteco (PRG) sind in ihren Reformbemühungen gescheitert. Der Antiimperialismus hat sich in propagandistischen Äußerungen erschöpft, die Abhängigkeit aber nicht verringert. Reformen sind in den Anfängen steckengeblieben, Bolivien ausgenommen ${ }^{25}$. Dem Viel-Klassen-Charakter dieser Parteien entsprechend haben sie politische Kompromisse nach allen Seiten geschlossen, ohne dadurch den anfangs gesteckten politischen Zielen entscheidend näher gekommen zu sein. Gescheitert sind sie u. a. auch dadurch, daß die traditionellen Parteien Lateinamerikas, die Konservativen, Liberalen und Radikalen, Teile der Forderungen nach sozio-ökonomischen Veränderungen in ihr eigenes Programm übernommen haben. Die populistischen Parteien haben sich nicht intensiv genug mit vordringlichen Reformen, wie z. B. mit der Agrarreform und der Arbeitsplatzbeschaffung, beschäftigt und sich dadurch von den übrigen Parteien abgesetzt.

\footnotetext{
18 Vgl. die Aufstellung populistischer Parteien von Lamberg, a. a. O., S. 15, der sie allerdings auf die demokratischen populistischen Parteien einschränkt. - Umfassender in: H. Kantor, "Los Partidos Populares in America Latina “, in: Journal of Inter-American Studies, 6 (1964), S. 222.

19 A. Hennessy, "Latin America «, in Ionescu/Gellner Populism, a. a. O., S. 28-61.

20 Parteien in Lateinamerika, a. a. O., S. $140 \mathrm{ff}$.

21 Ibid., S. 143.

22 Ibid., S. 144, insbesondere die Aufzählung dieser Parteien, die vom Autor für die sechziger Jahre zusammengestellt wurde.

23 Ibid.

24 Los Partidos Populares a. a. O., S. 232.

25 Lindenberg, a. a. I., S. 145.
} 


\section{Hauptmerkmale des Populismus}

Bis heute gibt es keine allgemein akzeptierte Theorie des Populismus. In meinen Ausführungen stütze ich mich hauptsächlich auf die Thesen eines der wichtigsten Theoretiker, auf Torcuato S. Di Tella ${ }^{26}$.

Di Tella sieht als Basis des Populismus die Masse der mit ihrer jetzigen sozialen und politischen Lage Unzufriedenen. Sie bilden eine „disposable mass"27 (verfügbare Masse), die sich leicht für ihre Interessen einsetzen läßt. Die Erwartungen dieser Masse richten sich dabei nach dem, was vergleichbare Gruppen der Gesellschaft westlicher Industriestaaten bereits erreicht haben. Die dringendste Forderung ist ein Ar" $^{\text {reitsplatz }}{ }^{23}$.

Ein bed' tsames Moment bei der Definition des Populismus ist die „Anti-Statusquo-Tendenz"29. Diese Tendenz verstärkt sich immer dann, wenn wirtschaftliche oder politische Schwierigkeiten auftreten. Ihr Ausdruck ist Unzufriedenheit breiter Bevölkerungsschichten, die sich aus Personen und Gruppen aller sozialen und politischen Schattierungen zusammensetzen. Die Gemeinsamkeit der Status-QuoKritik vereint sie um so leichter in einer populistischen Bewegung. Bei Slumbewohnern von Großstädten und armen campesinos ist sie meist noch nicht bewußt vorhanden. Sie wird ihnen oft erst von politischen Gruppen und Intellektuellen ins Bewußtsein gerufen.

Die „disposable mass" und die aus der oberen- und mittleren Mittelschicht stammende "Führungselite" 30 sind zwei Hauptelemente, die als Grundlagen populistischer Stärke von Di Tella genannt werden. In der Führung sind Militärs, Geschäftsleute, aber auch geistliche Würdenträger. In der Regel aber sind es vom System enttäuschte Intellektuelle und auf Reform bedachte Studenten ${ }^{31}$. Die Intellektuellen empfinden sich als überflüssig in einem System, das ihre Arbeitskraft nicht braucht und ihnen keine ihrer Ausbildung entsprechende Privilegien einräumt ${ }^{32}$. Aus der Führungselite ragt oft der "charismatische Führer" ${ }^{\text {"33 }}$ heraus und ist die bestimmende Figur. Die politisch unerfahrenen Massen brauchen diesen Führer, der ihnen die Komplexität des politischen Lebens in verständlicher Form nahebringt $\mathrm{t}^{34}$. Dies geht historisch auf den „personalismo“" 35 zurück.

Das dritte Grundelement, die „Ideologie“, ist die entscheidende Verbindung zwischen Führung und Parteibasis. Aus vagen programmatischen Zielen zusammengesetzt, dient sie dem Hauptzweck, einen kollektiven Enthusiasmus zu schaffen. Diese Ideologie kann nach rechts oder links ausgerichtet sein, da sie aber dem „Viel-Klassen-Charakter" 36 der Basis entsprechen muß, enthält sie Elemente beider Richtungen, ohne sich für eine festzulegen. Sie wird von „einem Bündel grundlegender sozialer Forderungen" ${ }^{37}$ zusammengehalten.

\footnotetext{
26 Populism and Reform, a. a. O., S. 47-74.

27 A. Hennessy, a. a. O., S. 29.

28 A. Angell, "Party Systems in Latin America “, in: Political Quarterly, London, 37 (1966), S. 316.

$29 \mathrm{Vgl}$. Di Tella, a. a. O., S. 50: "The incongruent groups (generally of above-average status) and the mobilized disposable masses complement each other. Their social situations are different, but what they have in common is passionate hatred of the status quo."

30 Angell, a. a. O., S. 315.

31 Ibid.

$32 \mathrm{Di}$ Tella spricht hier von „status incongruence“, a. a. O., S. $50 \mathrm{ff}$; Angell, a. a. O. S. 315. 33 Vgl. "Politik als Beruf ", in: M. Weber, Gesammelte Politische Schriften (Hrsg. J. Winckelmann),

34 Angell, a. a. O., S. 317

35 Die Form der persönlichen Beziehung zwischen nichtgebildeten Bevölkerungsschichten und ihrem Vorgesetzten, Herrn, Führer etc. Vgl. J. Lambert, Latin America, Berkeley 1967, S. 359-362.

36 Vgl. Di Tella, a. a. O., S. 57 ff., das Kapitel „Multi-Class, Integrative Parties“; sowie Tabelle 2, S. 57.

37 Angell, Party Systems, a. a. O., S. 318.
} 
Der Nationalismus populistischer Bewegungen versteckt sich hinter der Verteidigung ausgebeuteter Massen, deren Ausbeuter in der einheimischen Oligarchie und in ausländischen Kapitalgesellschaften, besonders amerikanischen, zu finden sind ${ }^{38}$.

\section{Die ANAPO als populistische Partei}

Die erste populistische Bewegung in Kolumbien war die unter dem linksliberalen Führer Gaitán. Er hat es als erster verstanden, die Massen zu mobilisieren und ihnen ein Gefühl von politischer Macht zu vermitteln. Für Gaitán war die Verbesserung ihrer sozialen Lage sein erklärtes Ziel ${ }^{39}$. Er identifizierte sich mit den Massen und sah sich als eins mit ihnen:

„I am.not a man, I am a people“40.

Als Gaitán 1948 auf offener Straße ermordet wurde, gerieten die Massen außer Kontrolle und versetzten der Ober- und Mittelschicht einen empfindlichen Schock. Führerlos, haltlos in Wut und $\mathrm{Haß}$ gegenüber den angeblich Verantwortlichen, konnten sie nur mit Gewalt antworten. Die Masse wurde zum blinden Mob, der vor nichts, nicht einmal vor Brandschatzungen von Kirchen, zurückschreckte. Die "disposable mass" braucht also, damals wie heute, den charismatischen Führer, der ihre Interessen gegenüber den oberen Schichten vertritt, gleichzeitig aber die Emotionalität und soziale Unzufriedenheit der Masse lenkt.

Rojas Pinilla versteht die ANAPO als nationalistisch, revolutionär und populistisch $^{41}$. Es ist hier zu untersuchen, ob die im vorherigen Kapitel aufgestellten Kriterien des Populismus für die ANAPO zutreffen.

Rojas Pinilla steht seit fast einem Jahrzehnt an der Spitze der ANAPO. Er ist der charismatische Führer, dem die Massen vertrauen und der ihrem traditionellen "patrón" 42 entspricht. Denn obwohl die Marginalen in den Städten oder am Rande der Großstädte wohnen, legen sie ihre ländlichen Gewohnheiten und Traditionen nur sehr langsam ab. Rojas Pinilla verspricht, sich für großzügige soziale Verbesserungen einzusetzen ${ }^{43}$. Er kommt nicht aus ihrer Schicht, sondern aus der oberen Mittelschicht und dürfte heute aufgrund seines sich angeeigneten Vermögens zur Oberschicht gezählt werden. Demgemäß gehört er zur Führungselite.

Die „disposable mass" als Hauptanhängerschaft der ANAPO rekrutiert sich aus den Marginalen der Großstädte ${ }^{44}$. Aber auch stark bevölkerte Departements wie Valle, Antioquia, Boyacá und Santander haben bei den Wahlen 1970 überwiegend für Rojas Pinilla und die ANAPO gestimmt ${ }^{45}$.

\footnotetext{
38 J. Lambert, Latin America, Berkeley 1967, S. 204; vgl. im besonderen: Plataforma Ideologica y Politica de Alianza Nacional Popular, Partido del Pueblo, Villa de Leyva 1971, S. 1-2.

39 Vgl. Dix, a. a. O., S. 102, der die konkreten Programmpunkte nennt.

40 Ibid, S. 101 .

41 Plataforma, S. 1.

42 ,. - means, protector' as well as ,landlord', in: P. G. Snow, Polotical Forces in Argentina, Boston 1971 , S. 14.

43 Reportaje.de J. E. Pulido/J. E. Orduz, Habla el General Rojas Pinilla „El Pueblo Escogió a María Eugenia“, in: El Periodico, 10. Juli 1972.

44 Marco Palacios weist aufgrund nationaler Statistiken nach, daß die ANAPO in den Städten Bogotá und Medellin allein von den unteren Schichten, ,estrato bajo“, 62\% und 64\% aller abgegebenen Stimmen in den Präsidentschaftswahlen im Jahr 1970 erhielt: „El Populismo en Colombia, Bogotá 1971, S. 89.

45 Sepúlveda Niño, Elites Colombianas en Crisis, o. O."1970, S. 119.
} 
Rojas Pinilla hat in Reden und Interviews mit Zeitungen wiederholt seine Person in den Hintergrund gestellt, aber die Rolle des Volkes in Anlehnung an Worte Gaitán's als wichtigstes Fundament seiner Bewegung herausgehoben.

„El pueblo es superior a sus dirigentes" ${ }^{46}$.

Die Ideologie als Ideengerüst politischer Handlungen und Ziele der ANAPO entspricht in ihrem „Viel-Klassen-Charakter" und ihrer vagen Programmatik den Kriterien des Populismus. Die Ideologie wurde erstmals schriftlich im Programm „el Decálogo"47 fixiert, das aus zehn Punkten als Grundlage politischer Aktion bestand. Bei der offiziellen Gründung der Partei 1971 wurde dann eine sehr ausführliche Parteiplattform vorgelegt, die „Plataforma Ideologica y Politica de ANAPO“.

In der Parteiplattform wird zwar die Oligarchie der Parteien-Koalition der Immoralität und der Ausbeutung der unteren Volksmassen bezichtigt, ihr aber nicht der offene Kampf angesagt ${ }^{43}$. Die populistische Ideologie bestätigt sich auch in den "grundlegenden sozialen Mindestforderungen" für die unteren Schichten. Sie sollen nicht nur besser wohnen und einer ständigen Arbeit nachgehen können, sondern auch freie Erziehung und freie medizinische Versorgung genießen ${ }^{40}$. Den oberen Schichten wird aber eine weitgehende Mithilfe und Beteiligung, besonders im finanziellen Bereich, bei der Entwicklung des Landes angeboten ${ }^{50}$. Die Ideologie ist stark auf die unteren Klassen ausgerichtet, vermeidet aber die offene Konfrontation mit den besitzenden Klassen. Es wird eine gelenkte Staatswirtschaft gefordert, aber keine Nationalisierung von privatem Kapital oder Besitz. Der „Viel-Klassen-Charakter" der Ideologie wird weitgehend gewahrt.

Der uneinheitliche, vielseitige Charakter des Parteienprogramms wird auch in einem so bedeutenden Programmpunkt wie der Agrarreform deutlich.

Noch immer leben 45 Prozent der kolumbianischen Bevölkerung (im Jahre 1970) in ländlichen Gebieten, von denen der größte Teil kein eigenes Land besitzt ${ }^{51}$.

Die Kritik der Parteiplattform wendet sich vor allem gegen die geringe Verteilung an Land. Es wird behauptet, daß von 1970 bis Juni 1971 nur insgesamt 70000 Hektar zur Verteilung an 5347 kleine Bauern kamen. Die Zahl der campesinos, die einen echten Bedarf an Land vorweisen können, beträgt aber 850000 , die 550000 Minifundien-Besitzer nicht mitgerechnet ${ }^{52}$.

Ein Senatsbericht des US-Kongresses vermerkt, daß zwischen 1961 und 1967 nur 54000 Landbesitztitel aus der Gesamtzahl von 400000 oder 500000 landloser Familien vergeben wurden ${ }^{53}$.

Das Ziel einer von der ANAPO vorgeschlagenen Landreform ist die umfangreiche Neuverteilung des nicht genutzten Latifundienbesitzes und die Gründung von Kooperativen ${ }^{54}$. Es sollen lebensfähige und wirtschaftliche kleine Bauernhöfe -

\footnotetext{
46 „El Pueblo .... , in: El Periodico, 10. Juli 1972.

47 Niño, a. a. O., S. 169.

48 Plataforma, S. 1.

49 Ibid., S. $21-24$.

50 Ibid., S. 12, 14.

51 L. Schoultz, „Urbanization and Changing Voting Patterns: Colombia 1946-1970, in. Political 54 L. Schoultz, "Urbanization and Changing Voting Patterns: Colombia 1946-1970, in: Political Science Quaterly, 87 Jg., No. 1, March 1972, S. 29, Tabelle 2 - Alle Personen leben in Dörfern mit etwas

52 Plataforma, S. 26; B. Lefoudre, „Le Gouvernement Présente un Bilan . . . in: Le Monde, 26. November 1970.

53 "Colombia: A. Case History of U.S. Aid“, in: P. E. Sigmund, Models of Political Change in Latin America, New York 1970, S. 281; vgl. auch: H. Kantor, Patterns of Politics and Political Systems in Latin America, Chicago 1969, S. 403

54 Plataforma, S. 27.
} 
aber keine Minifundien - entstehen. Die so gesteigerte Produktivität der Landwirtschaft soll die nationale Wirtschaft wieder unabhängiger von fremden Einfuhren machen. Die ANAPO fordert nicht mehr und nicht weniger als die INCORA ${ }^{55}$, die als paritätisch besetzte und bevollmächtigte Organisation der beiden Parteien sowie der betroffenen Schichten für die Realisierung der Gesetze 135 von 1961 und 1 von 1968 einzutreten hat. Gesetz 135 besagt u. a., daß die Teilung eines Grundstückes in weniger als 3 Hektar unzulässig und ungesetzlich ist $^{56}$.

In einem neueren Resümee der INCORA werden die Errichtung von Kooperativen in der Landwirtschaft und im Handel sowie mehr Kredite gefordert ${ }^{57}$. Die neuen revolutionären Ansätze der ANAPO beschränken sich auf Wiederholungen der Forderungen, wie sie die Frente Nacional selbst gestellt hat, oder auf revolutionär klingende Sätze ${ }^{53}$. Die Forderung der ANAPO nach der Neuverteilung von nicht genutztem Latifundienbesitz wird dadurch sekundär, daß bekannt und bewiesen ist, daß das nicht genutzte Latifundienland wenig produktiv und in der Regel felsig ist. Nur die Verteilung von bereits bebautem oder fruchtbarem Boden kann für die landlosen Bauern eine echte Existenzgrundlage werden.

\section{Die Wahlerfolge der ANAPO}

In einem Rückblick 1970 bemerkte der Korrespondent der NZZ: „Anfang der

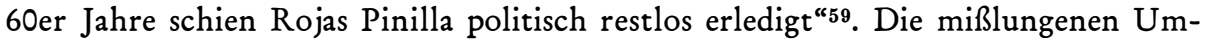
sturzversuche nach seiner Rückkehr aus dem Exil, die seine Urheberschaft sehr deutlich gezeigt hatten, waren ein sicheres Indiz dafür. Doch in dem Maße, in dem er selbst untragbar geworden war, legte er alle organisatorischen Aufgaben, die den Aufbau einer eigenen Parteiorganisation erforderten, in die Hände seiner politisch aktiven Tochter María Eugenia.

In den Präsidentschaftswahlen 1962 hatten sich vier Kandidaten aufstellen lassen. Guillermo León Valencia war der offizielle Kandidat der Frente Nacional, der mit 1647000 Stimmen 62 Prozent aller abgegebenen Stimmen auf sich vereinigen konnte. Rojas hatte nur 55000 Stimmen erhalten, was 2,1 Prozent der Gesamtstimmenzahl entsprach ${ }^{60}$. Als Test für ein politisches comeback war es ein bescheidenes Ergebnis. Die Stimmen für ihn wurden zwar gezählt, aber als ungültig erklärt, weil $\mathrm{ihm}$ die politischen Rechte noch nicht wieder zuerkannt worden waren ${ }^{61}$. Ahnlich war es López Michelsen ergangen, dem Führer der oppositionellen linksliberalen Gruppe der Liberalen Partei, Movimiento Revolucionario Liberal (MRL). López Michelsen sorgte für eine beachtliche Stimmenzersplitterung. Mit 524000 Stimmen oder 23,7 Prozent der abgegebenen Stimmen hatte er den zweiten Platz nach dem siegreichen konservativen Kandidaten Guillermo León Valencia belegen können ${ }^{62}$. Beide abgewiesenen Kandidaten waren sich in der konsequenten Ablehnung des Koalitionssystems einig gewesen.

\footnotetext{
55 Instituto Colombiano de Reforma Agraria; vgl. für die ersten Jahre der INCORA das Kapitel 5, in: P. M. Holt Colombia and Tomorrow, New York 1964, S. 74-100.

56 Dix, a. a. O., S. 156.

57 "Colombia: Plan de Acción de la Alianza Liberal-Conservadora“, in: Documentos, Caracas 1969, Nr. 39, S. 162 .

58 Plataforma, S. 26

59 "Der knappe Wahlsieg Pastranas in Kolumbien ${ }^{\alpha}$, in: Neue Zürcher Zeitung (NZZ), 5. Mai 1970.

60 Holt, a. a. O., S. 58.

61 Schoultz, a. a. O., S. 39, Tabelle 4, Fußnote.

62 Holt, a. a. O., S. 58.
} 
Der Verlust von fast 580000 Stimmen bei den Präsidentschaftswahlen 1962 charakterisiert auffällig die bereits früh einsetzende Ablehnung des politischen Systems der "Frente Nacional“ durch die Wähler. Die Abgabe dieser Stimmen kann nur als Protest gewertet werden, da von vornherein feststand, daß aufgrund der „Frente Nacional“-Bestimmungen nur offiziell nominierte Kandidaten dieser Koalition Präsident werden konnten.

Die Parlamentswahlen des Jahres 1962 hatten für Rojas Pinilla und seine Anhänger einen ersten bescheidenen Anfang bedeutet. Sie gewannen 6 Sitze im Repräsentantenhaus und 2 im Senat. 1964 konnte der Sitzanteil im Repräsentantenhaus auf 27 erhöht werden. Erstmals warb Rojas Pinilla auch auf liberaler Seite und gewann einen liberalen Sitz im Repräsentantenhaus ${ }^{63}$. Die Absicht war, möglichst viele Wählerstimmen abzufangen. Von 1962 bis 1964 erhöhte sich die Gesamtstimmenzahl für die Rojas-Gruppen von 4 Prozent auf 18 Prozent $^{64}$. Die Gesamtstimmenabgabe war in der gleichen Zeit von 3,1 Mill. im Jahre 1962 auf 2,2 Mill. 1964 gesunken, obwohl die Zahl der Wahlberechtigten um 6 Mill. angewachsen war ${ }^{65}$. Der Stimmenzuwachs für die koalitionsfeindlichen Rojas-Gruppen und die gleichzeitig zurückgehende Wahlbeteiligung waren ein Alarmzeichen für die „Frente Nacional", die von nun ab soziale Reformen und wirtschaftliches Wachstum stärker in den Vordergrund stellte.

1966 war wiederum ein Wahljahr für Parlaments- und Präsidentschaftswahlen. Der langsame Zuwachs von Stimmen für die Rojas-Gruppen setzte sich fort.

In den Präsidentschaftswahlen war aufgrund der üblichen Ämterrotation ein Liberaler zu wählen ${ }^{66}$. Es standen sich zwei Kandidaten aus dem liberalen Lager gegenüber. Lleras Restrepo war der offiziell von der „Frente Nacional“ nominierte Kandidat und José Jaramillo Giraldo vertrat die politischen Interessen der beiden Rojas-Flügel. Giraldo hatte von Anfang an keine Chance, den äußerst populären Führer der Frente Nacional zu besiegen. Aber dem im Hintergrund agierenden Führer, Rojas Pinilla, ging es auch nur darum, die bis dahin eindeutige Führung der Koalitionsregierung in Frage zu stellen. Die Chance für einen Sieg über die Frente Nacional war allein schon deshalb gering, weil es im ganzen Land noch keine umfassende Parteiorganisation gab. Von 1962 bis 1966 konnten RojasGruppen nur in den großen Städten erfolgreiche Wahlkampagnen durchführen ${ }^{67}$, dabei konzentrierten sie sich auf die marginale Stadtbevölkerung. In diesen Gebieten, besonders in Bogotá, konnte Giraldo die meisten Stimmen für sich gewinnen. Das ist um so bedeutender, weil er nur geringe Popularität und politische Ausstrahlungskraft besaß. 741203 der nationalen Gegenstimmen oder 28,27 Prozent entfielen auf ihn, während sein Gegner, Lleras Restrepo mit 1881502 Stimmen oder 71,73 Prozent als klarer Sieger hervorging ${ }^{63}$.

In den wenig später folgenden Parlamentswahlen erhielten die konservativen- und liberalen Rojas-Gruppen 36 Sitze im Repräsentantenhaus und 15 im Senat. Bemerkenswert war, daß die 15 Senatssitze nur vom liberalen Flügel gewonnen worden waren. Gemessen an der Gesamtzahl von derzeit 190 Sitzen im Repräsentan-

\footnotetext{
63 Dix, a. a. O., S. 114, 282.

64 Ibid., S. 282.

65 Holt, a. a. O., S. 59 - Eine ausführliche Analyse des Stimmenrückgangs in den 60er Jahren gibt: Gilhodes, L'Abstention, S. 57.

66 Eine ausführliche Beschreibung des Systems der Frente Nacional gibt: Dix, a. a. O., S. 134-136.

67 Schoultz, a. a. O., S. 37.

68 Ibid., S. 39, Tabelle 4; vgl. auch: Dix, a. a. O., S. 146, Tabelle 7.
} 
tenhaus war das ein Anteil von 17 Prozent und bei den 106 Sitzen im Senat von 14 Prozent ${ }^{69}$.

Der Gewinn an Stimmen und Kongreßsitzen war von 1962-1966 kontinuierlich fortgeschritten, ohne daß dies schon eine Bedrohung für die Regierung der Frente Nacional darstellen konnte. Die Rojas-Gruppen stellten nur die stärkste Oppositionsgruppe innerhalb der Konservativen Partei und eine schwache innerhalb der Liberalen Partei dar. Die übrigen Gruppen der Opposition rekrutieren sich aus den oppositionellen Liberalen, „des von dem Philokommunisten bis zu den Linksliberalen reichenden Movimiento Revolucionario Liberal (MRL)"70, und der Alvarista-Gruppe der Konservativen Partei. Die zuletzt genannte wurde von dem Sohn des ehemaligen konservativen Präsidenten Laureano Gómez, Alvaro Gómez Hurtado, angeführt und galt als rechtskonservativ. Die drei Gruppen der Opposition verfügten zusammen über 83 Sitze im Repräsentantenhaus und über $39 \mathrm{im}$ Senat. In beiden Häusern besaßen sie jetzt mehr als ein Drittel der gesamten Sitze und hatten somit die Möglichkeit, neue Gesetze etc., für die eine Zustimmung von Zweidrittel der Mitglieder notwendig war, durch gemeinsame Stimmenverweigerung zu verhindern. Eine erste Probe gab die neue Opposition nach der Einberufung des Parlaments nach den Wahlen. Sie verweigerte ihre Stimmen bei der Wahl des Vizepräsidenten und des Präsidenten des Parlaments und führte später durch fortgesetzte Obstruktion in der Parlamentsarbeit eine ernste Regierungskrise herbei ${ }^{71}$. Die Einigkeit der Opposition war aber nur sporadich zu erreichen und das vordergründige Eigeninteresse jeder einzelnen Gruppe blieb erhalten. So gelang es der Liberalen Partei, das von seinen extrem linken Anhängern gesäuberte MRL ${ }^{72}$ kurz vor den Parlamentswahlen 1968 wieder in die Mutterpartei zu integrieren. Die Einigkeit dauerte nur bis Mai 1971, dann zerfiel die Liberale Partei wieder in zwei sich befehdende Lager von Links- und Rechtsliberalen. - Die Rojas-Gruppen schöpften alle Möglichkeiten, die das System der Frente Nacional oppositionellen Gruppen ließ, voll aus und trugen zu einer teilweisen Lahmlegung der Parlamentsarbeit bei.

1970 war wiederum ein Wahljahr für die Parlaments- und Präsidentenneubesetzung. Außerdem sollte dies die letzte Legislaturperiode unter dem Koalitionssystem sein, das 1974 offiziell endet. Erstmals in der kolumbianischen Geschichte wurden Parlaments- und Präsidentschaftswahlen auf einen Tag (19. April) gelegt, was mit einem erheblichen Sicherheitsrisiko für die Bevölkerung und einem ungeheuren organisatorischen Aufwand verbunden war.

In der Präsidentschaftswahl standen sich vier konkurrierende Kandidaten der Konservativen Partei gegenüber: Misael Pastrana, ein wenig bekannter, politisch farbloser Rechtsanwalt, als bevorzugter Kandidat des loyalen Frente Nacional-Flügels, Sourdis, Betancourt und der radikale Rojas Pinilla, inzwischen siebzigjährig und mit angegriffener Gesundheit. Pastrana war der aus zwei Parteitagen der Konservativen Partei hervorgegangene offizielle Kandidat der Partei, der auch die notwen-

$69 \mathrm{R}$. H. McDonald, "Political Parties in Colombia and Venezuela Contrasts in Substance and Style“, in: The Western Political Quaterly, 18 (1965) 1, S. 18, Tabelle 4

70 „Die Neubestellung des kolumbianischen Parlaments“, in: NZZ, 26. März 1966.

71 Der liberale Präsident Lleras Restrepo drohte mit dem Rücktritt, als er sein Reformprogramm durch die Opposition gefährdet sah. Außerdem war es ihm nicht gelungen, eine Verfassungsänderung durchzusetzen, um die notwendige Zweidrittelmehrheit bei Parlamentsbeschlüssen durch die einfache Mehrheit zu ersetzen.

72 "Aufschwung in Kolumbien“, in: NZZ, 25. Oktober 1968

73 Die Koalition durfte nur einen gemeinsamen Kandidaten für beide Parteien aufstellen. 
dige Zustimmung der Liberalen Partei gefunden hatte ${ }^{73}$. Die auf den Parteitagen unterlegenen Kandidaten „widersetzten sich aber der Parteidisziplin und zogen auf eigene Faust in den Wahlkampf "74.

Sah es bei der Stimmenzählung vorerst so aus, als ob Rojas Pinilla gewinnen würde, so ging am Ende der Auszählung Misael Pastrana als Sieger hervor. Der Sieg war allerdings äußerst knapp gewesen und bis heute deutet vieles darauf hin, daß Rojas' Behauptung von einer Wahlfälschung nicht ohne Recht besteht ${ }^{75}$. Pastrana hatte insgesamt 1625025 Stimmen erhalten, Rojas Pinilla hingegen $1561468^{76}$. Nach der offiziellen Zählung war er also nur mit 63557 Stimmen unterlegen.

\section{Die Departementswahlen 1972}

Die Wahlen für die einkammerigen Provinzparlamente und die Gemeinderäte werden als Barometer für die entscheidenderen Kongreß- und Präsidentschaftswahlen angesehen. 1970 hatte die ANAPO in diesen Wahlen 1,3 Mill. Stimmen gewonnen und damit die Kontrolle über 9 der 22 Provinzparlamente und 400 Gemeinderäte erhalten. 1972 mußte sie völlig unerwartet einen Stimmenverlust von 850000 hinnehmen und verlor damit die Mehrheit in allen Provinzparlamenten und in 90 Prozent der vorher von ihr kontrollierten Gemeinderäten ${ }^{77}$. Die große Gewinnerin war die Liberale Partei, die mit 1,3 Mill. Stimmen 400000 mehr als die Konservative Partei erhielt ${ }^{78}$. Der Stimmenrückgang ist mit der schlechten Arbeit der ANAPO in den Provinzparlamenten und Gemeinderäten, wie auch mit dem Streit innerhalb der Partei, der nach dem Austritt von Nacho Vives 1970 immer wieder aufgeflackert ist, zu erklären.

\section{Die Wahlen vom 21. April 1974}

Die Präsidentschafts- und Parlamentswahlen des Jahres 1974 waren deshalb von besonderen Bedeutung, weil in diesem Jahr die Koalitionsvereinbarung der beiden Parteien auslief und sich erstmals nach 16 Jahren alle Parteien zur Wahl stellen konnten. Allerdings war bereits vor diesem Termin die Verlängerung der Koalition auf lokaler und departementaler Ebene von beiden Parteien beschlossen worden. Nach dem Sieg der Liberalen Partei in diesen Wahlen hat die Partei erklärt, daß sie auch im Parlament weiterhin die Sitze mit den Konservativen teilen werde, die Nationale Front also auch ohne offizielle Verträge fortgesetzt wird.

Der Sieg der Liberalen Partei und ihres Präsidentschaftskandidaten López Michelsen, der in den sechziger Jahren den linken Flügel dieser Partei führte und heute zur Mitte gehört, war allgemein erwartet worden. López' Sieg mit 2535374 Stimmen war eindeutig, denn die Differenz zum nächsten Kandidaten, dem Konservativen Gómez Hurtado, der 1467845 Stimmen erhalten hatte, betrug

74 "Der knappe Wahlsieg Pastranas in Kolumbien“, in: NZZ, 5. Mai 1970.

75 Rojas'. Anschuldigungen führten zu einer 2. Zählung, die allerdings die Ergebnisse der ersten nur

76 Schoultz, a. a. O., S. 39, Tabelle 4, dieser bezieht sich auf die offizielle Quelle: Registraduria Nacional del Estado Civil, unpublished electroral data 1970.

77 „Zerstrittene Liberale als Wahlsieger in Kolumbien ${ }^{\alpha}$, in: NZZ, 14. April 1972.

78 "Sieg der Liberalen“, in: Die Zeit, 12. Mai 1972. 
1067 845. María Eugenia, die Präsidentschaftskandidatin der ANAPO und erste Frau als Anwärterin auf das Präsidentenamt, folgte erst an dritter Stelle mit 450143 Stimmen. Der gemeinsame Kandidat der Kommunisten und weiterer linksextremer Parteien, Unión Nacional de Oposición (UNO), Escheverri Mejia, konnte nur 123459 und der Kandidat der Christdemokraten, Hermes Duarte, 5703 Stimmen auf sich vereinigen ${ }^{79}$.

Die hohen Erwartungen, die María Eugenia und die ANAPO an diese Wahlen gestellt hatten, sind bitter enttäuscht worden. Die Folge ist, daß die ANAPO nicht mehr den Anspruch einer Massenpartei erheben kann und ihre Vertretung nur noch in wenigen Departements und Gemeinden gesichert ist. Damit ist der Abstieg dieser Partei vom Fast-Sieg in den Wahlen des Jahres 1970 über den großen Stimmenverlust in den Departementswahlen des Jahres 1972, in denen sie nur noch um 800000 Stimmen erhielt, und die nochmalige Halbierung dieses Stimmenanteils in den Wahlen dieses Jahres, unmißverständlich vorgezeichnet. Von den 321 Kongreßsitzen, von denen sie seit den letzten Parlamentswahlen 1970110 besetzt hatte, hat sie jetzt nur noch 2980 .

Für den nicht unerwartet eingetretenen Mißerfolg der ANAPO lassen sich mehrere Gründe anführen:

1. Der große Wahlerfolg der Liberalen Partei fiel nicht zufällig mit einer selten erreichten Einheit innerhalb der Partei zusammen. López Michelsen, der noch bis 1967 mit dem linken Flügel der Partei, das MRL, eine Vielzahl der Stimmen angezogen hatte, sorgte nun persönlich für die Einheit der Partei. In der ANAPO vollzog sich aber gerade der umgekehrte Vorgang. Die in den sechziger Jahren im Aufbau begriffene Partei hatte sich stets um die Einheit der in der Liberalen und Konservativen Partei operierenden Flügel der ANAPO bemüht. Für Kooperation und Integration innerhalb der Partei hatte sich Rojas Pinilla eingesetzt, der auch nach allen Seiten geschickt taktieren konnte. In der Familie von Rojas Pinilla waren oft unterschiedliche politische Standpunkte zwischen Vater und Tochter aufeinandergestoßen, denn María Eugenia trat meist für radikalere Lösungen ein. Und gerade aus dieser Familie wurde nach dem politischen Rücktritt von Rojas die Spaltung betrieben. Einige Monate vor den Wahlen dieses Jahres trat María's Bruder mit einem Flügel der ANAPO zur Liberalen Partei über. Innerparteilich geschwächt ging daher die ANAPO in die entscheidenden Wahlen dieses Jahres.

2. Die Aufstellung einer Frau als Präsidentschaftskandidatin war ein Wagnis von Anfang an und wäre es zweifellos auch für die große Liberale Partei wie für jede andere gewesen. Denn gerade in diesem von Traditionen beherrschten Land kann eine Frau noch nicht jeden angestammten Platz des Mannes einnehmen. Selbst in den als fortschrittlich geltenden westlichen Demokratien haben immer noch Männer die politisch entscheidenden Ämter inne. - Ihre durch alle Teile des Landes unternommene Wahlkampagne konnte das Mißtrauen gegenüber ihrer politischen Qualifikation nur geringfügig abbauen.

3. Die Abgabe vieler Stimmen in den Wahlen 1970 für die ANAPO ist als Proteststimmabgabe gegen die "eingefrorene Demokratie“ der Nationalen Front zu sehen. Nach der teilweisen Beendigung der Aimterteilung auf allen parlamentarischen Ebenen und der Rotation des Präsidenten zwischen den beiden Parteien konnte der Wähler diesmal die Stimme dem Kandidaten seiner Wahl geben.

79 Alle Zahlenangaben aus: „Oficialmente, la Mayoría de López Pasa del Millón“, in: El Espectador, 24. April 1974.

$80{ }_{55 \%} \%$ del Congreso en poder del liberalismo“, in: El Tiempo, 23. April 1974. 
4. Eine deutliche Stimmenverlagerung der marginalen Bevölkerung der Großstädte zu den beiden traditionellen Parteien ist zu erkennen. In den Wahlen 1970 hatte die ANAPO eine Mehrheit der Stimmen aus der Unterschicht und der unteren Mittelschicht fast aller Großstädte erhalten ${ }^{81}$. Das Beispiel der Hauptstadt Bogotá als eine ihrer Hochburgen zeigt, daß die ANAPO mit 53284 Stimmen weit hinter Liberale $\left(298\right.$ 028) und Konservative (81 352) zurückgefallen ist ${ }^{82}$. Bestätigt wird dies durch die im Senat und Repräsentantenhaus gewonnenen Sitze für die Provinz Cundinamarca, in der als gesonderte administrative Einheit auch Bogotá liegt. Von den 16 im Jahre 1970 erhaltenen Sitzen für beide Häuser des Kongresses erhielt die ANAPO 1974 nur ein Viertel, also 4 Sitze $^{83}$. Diese wenigen Zahlenbeispiele verdeutlichen, daß die ANAPO gerade in jenen Schichten an Stimmen verloren hat, auf die sie am meisten gebaut hatte und auf die in wesentlichen Punkten das Parteiprogramm und die Wahlplattform 1974 ausgerichtet waren.

War mit Sicherheit anzunehmen, daß ein Teil der ANAPO-Wähler, die 1970 zum großen Sieg der Partei beigetragen hatten, Protestwähler, Enttäuschte der $\mathrm{Na}$ tionalen Front waren, so zeigt doch, daß gerade die sozial benachteiligten Schichten heute keine entscheidenden Anderungen von der populistischen ANAPO mehr erwarten. Dabei muß nochmals darauf hingewiesen werden, daß die ANAPO in ihrem 12-Punkte-Wahlprogramm eine Einschränkung der Parteienoligarchie und die Partizipation breiterer Schichten in Industrie und Landwirtschaft gefordert hatte ${ }^{84}$, sich also ohne Einschränkung für alle in der Gesellschaft Benachteiligten einsetzen wollte. Eine Erklärung für die Hinwendung dieser Schichten zur Liberalen Partei läßt sich nur schwer finden.

$81 \mathrm{Vgl.} \mathrm{Niño,} \mathrm{a.} \mathrm{a.} \mathrm{O.,} \mathrm{S.} \mathrm{105,} 119$.

82 "Officialmente, la Mayoría de López Pasa del Millón ${ }^{\alpha}$, in: El Espectador, 24. April 1974.

83 Niño, S. 116-117, Tab. 1,2; „57\% del Congreso en poder . . . «, in: El Tiempo, 23 April 1974

84 H. Rolvering, „Das politische Kartell geht seinem Ende entgegen“, in: FAZ, 19. April 1974. 
By the Provisional Constitution Order a Parliamentary System was set up. The Parliament consisted of the members elected to the National Assembly and acted also as Constituent Assembly. It approved the Constitution on the 4th of November 1972. The main features of the Constituion are a chapter on principles of State policy which are based on four elements, namely nationalism, socialism, democracy and secularism. Fundamental rights are guaranteed including the right of property. Bangladesh is a parliamentary democracy with a Cabinet form of government. The Parliament is unicameral. The Constitution provides also an independent judiciary as guardian of the constitutional rights.

\section{Populism in Colombia: The ANAPO}

\section{By W. Wessel-Schulze}

Since independence there have been two dominant parties in Colombia: the Conservatives and the Liberals. For centuries each of them has governed the country until 1948. The following party strife, called the "violencia", has been one of the most violent and brutalized struggles of the twentieth century. At the brink of civil war and absolute chaos the military under the leadership of General Rojas Pinilla took over power. Being ousted by his colleagues in 1957 a military junta governed until August 1958. The party coalition of Conservatives and Liberals, called the "National Front", brought political stability with a system of parity and alternation. That meant equal share of all governmental offices and a change of the presidency between the two parties for sixteen years.

Having returned from exile Rojas Pinilla looked for supporters of a populist policy in the parties. Between 1960-1970 Rojas and his daughter, Maria Eugenia, were successful in building a mass party. In the 1970 presidential and governmental elections the so called ANAPO lacked only 65000 votes. In 1971 the ANAPO was officailly founded in the historic province city of Villa de Leyva.

The ANAPO has often been called a "populist party". according to Torcuato Di Tella the main elements of a populist movement or party are: a) a broad mass basis, b) an elite often dominated by a charismatic leader, c) a vague ideology. The mass basis of the ANAPO is recruited from the marginal population of the big cities. In the elections of 1970 the ANAPO received 60 to 65 per cent of all votes of the lower classes. In the leadership are men from the middle and upper classes. At its top stood Rojas Pinilla as the charismatic leader until 1972. Now his daughter holds this position. In the ideology, written down in the "plataforma", the dispossessed people are promised everything from land to free education. On the other hand capital or property of the upper class will not be nationalized, class-struggle is not forced.

In the elections of the province parliaments in 1972 the ANAPO has lost half of all votes compared with those won in the elections of 1970. In the presidential elections in April 1974 the ANAPO candidate, Maria Eugenia Rojas de Moreno, was the third with not more than 450000 votes. After this enormous loss of votes, especially in the lower classes, the fate of the ANAPO is unsecure. Surely it is not a competing third mass party any longer. 